\title{
Single Nanotube Voltammetry: Current Fluctuations Are Due to Physical Motion of the Nanotube
}

\author{
Hannah Hodson, ${ }^{\dagger}$ Xiuting Li, ${ }^{\dagger}$ Christopher Batchelor-McAuley, ${ }^{\dagger}$ Lidong Shao, ${ }^{\ddagger}$ \\ and Richard G. Compton ${ }^{*}$,
}

\begin{abstract}
${ }^{\dagger}$ Department of Chemistry, Physical and Theoretical Chemistry Laboratory, Oxford University, South Parks Road, Oxford OX1 3QZ, United Kingdom

*Shanghai Key Laboratory of Materials Protection and Advanced Materials in Electric Power, Shanghai University of Electric Power, 2103 Pingliang Road, Shanghai 200090, P.R. China
\end{abstract}

\section{Supporting Information}

ABSTRACT: Nanoimpacts of single palladium-coated carbon nanotubes on a gold substrate are studied to elucidate the origins of the fluctuation in the current-time response of the hydrogen oxidation reaction mediated at its surface. The chronoamperometric and cyclic voltammetric responses from a single nanotube immobilized on the gold surface were compared to analogous data on a carbon substrate to determine the possible influence of substrate material on the nanotube-electrode electrical contact. No significant distinction between the gold and carbon was found, indicating in light of the considerable differences in the substrate materials' intrinsic electronic structures that it is the nanomotion of a nanotube at the electrode surface which is likely responsible for the observed current modulation.

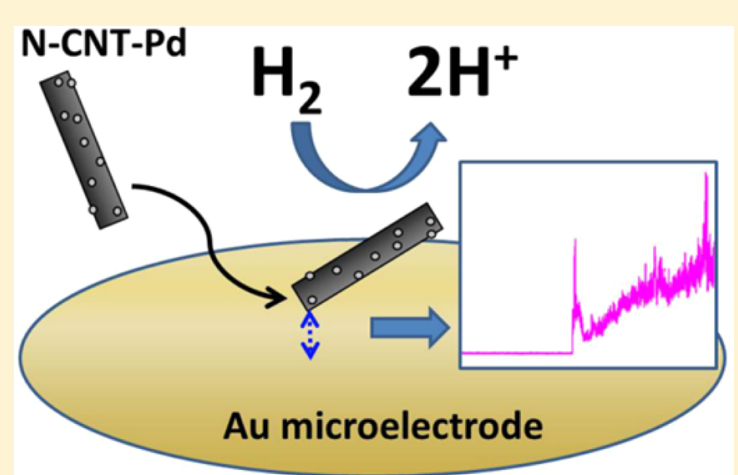
This nanomotion creates a varying contact resistance, to which the noise in the current-time signal of the mediated reaction is attributed. In addition, stochastic ex-situ adsorption of single nanotubes onto the gold electrode followed by careful drying of the electrode surface was found to drastically reduce the current fluctuation, again implying that a contact resistance arising from physical motion of the nanotube at the electrode is responsible for the modulation of current.

\section{INTRODUCTION}

The electrochemical detection and characterization of individual nanoparticles have received much attention. ${ }^{2-6}$ Broadly, these experimental studies either probe the direct electrochemical response of the nanoparticle itself ${ }^{7}$ or focus upon the mediation of a redox reaction at the nanoparticle surface, ${ }^{1,2,8}$ the objectives of both encompassing a diverse range of applications from fundamental to analytical or technological. $^{7-11}$ In the latter case of nanoparticle-mediated electron transfer, suspended nanoparticles catalytically active toward a redox species which is inert at the chosen electrode substrate stochastically collide with the electrode and mediate the reaction. This is observed as a "step on" in the current-time response, enduring for the duration of the nanoparticle's association with the electrode. Previous examples of these systems range from the initial studies on protonated $\mathrm{TiO}_{2}$ colloids ${ }^{12}$ to $\mathrm{Pt}$ nanoparticles which catalyzed proton reduction on impact with a carbon substrate; ${ }^{11} \mathrm{IrO}_{x}$ nanoparticles catalyzing the oxidation of water on impact with a Pt substrate ${ }^{8}$ and the reduction of hydrogen peroxide mediated at silver nanoparticles impacting on carbon. ${ }^{13}$ Recent work has shown the mediation of the hydrogen oxidation reaction (HOR) at carbon nanotubes modified with catalytically active $\mathrm{Pd}$ nanoparticles impacting a carbon fiber microwire electrode $(\mathrm{CFE}){ }^{1}$ it is this reaction which is further studied here.

Success in detecting nanoparticle-mediated redox activity is in part predicated upon the redox reaction of interest being significantly slower on the "inert" electrode substrate than on the nanoparticle surface of study. This large discrepancy in rates may be achieved either by the use of a suitable "inner-sphere" electron transfer reaction or by the insulation of the supporting electrode with a "thin" partially passivating layer. ${ }^{14,15}$ The use of carbon as a supporting electrode material is often beneficial due to the commonly associated sluggish heterogeneous electron transfer kinetics. ${ }^{16}$ Alternative metallic materials which often exhibit slow electron transfer kinetics, such as mercury, ${ }^{17-19}$ are complicated by the ready formation of chloride salts ${ }^{20}$ which can and do lead to erroneous results.

One prime aim of such mediated reaction studies is the ability to rapidly screen and study the structure-activity relationship of individual nanoparticles. Here a supporting electrode is immersed into a solution containing the nanoparticle of interest. Due to Brownian motion these particles

Received: January 21, 2016

Revised: February 18, 2016

Published: February 18, 2016 
randomly and stochastically collide with the supporting electrode. If a suitable potential is held at the supporting electrode, on impact a redox reaction may be driven at the surface of the nanoparticle. Assuming the nanoparticle is sufficiently conductive, the electrochemical reaction kinetics will likely be determined by the interfacial electron transfer: the rate of reaction may be limited either by the transfer of electrons between the nanoparticle and the supporting inert electrode, or at the nanoparticle/solution interface. Furthermore, as the nanoparticle approaches the potentiostated electrode from the solution phase the rate-determining step will likely vary as a function of the nanoparticle/electrode separation. At large separations the electron transfer from the nanoparticle to the electrode will be determined by the tunnelling probability; ${ }^{21}$ consequently, under this regime the rate of electron transfer will likely exhibit an exponential variation as a function of the nanoparticle/electrode distance. $^{21,22}$ For contacts with a diameter smaller than the mean free path of an electron, the interfacial resistance will scale as the inverse square of the contact radius (the so-called Sharvin limit corresponding to ballistic transfer across the interface); ${ }^{22}$ conversely for contacts larger than the electron mean free path, the transfer can be considered diffusive, and the interfacial resistance will be proportional to the inverse contact radius (Holm/Maxwell limit). ${ }^{23}$ Subsequently, at shorter distances and with the formation of an electrical contact, the interfacial rate of transfer may be sufficiently large for the ratedetermining step to switch to that of the nanoparticle/solution interface. However, the resistance of such a nanoparticle/ electrode contact will depend upon a variety of factors, not least the size of the contact area. For smaller, highly symmetric nanoparticles (spherical for example), it is reasonable that the nanoparticles' electrical contact with the electrode will be dominated by a single point. However, for larger and less symmetric particles the total current will constitute the summation of multiple parallel contributions. ${ }^{23}$

Such investigations into the activity of individual nanoparticles are also reliant on prolonged contact of the impacting nanoparticle with the electrode surface to allow the extraction of kinetic data; for instance a complete (forward then backward) cyclic voltammetric scan at $50 \mathrm{mV} \mathrm{s}^{-1}$ over a potential range of $2 \mathrm{~V}$ requires a residence time of at least $80 \mathrm{~s}$. Performing a variable scan rate study over the same potential range thus requires several minutes' contact. A workaround using current spike-type responses recorded at different potentials can be used to construct an effective "CV" from which kinetic insight may be gained. ${ }^{24}$ Other promising approaches involve nanoparticles immobilized by electric field attraction, ${ }^{25}$ Scanning electrochemical microscopy (SECM) mapping of a single nanoparticle in a prefabricated array, ${ }^{25,26}$ and the prior electrodeposition of a single nanoparticle, ${ }^{14,27,28}$ where in the latter two cases electrical contact is encouraged through the prior deposition of the nanoparticle onto the substrate. In particular Wain et al. demonstrate catalysis of the $\mathrm{HOR}$ at specific sites corresponding to agglomerated $\mathrm{Pt} / \mathrm{C}$ black nanoparticles, ${ }^{26}$ as well as a SECM resolution capable of distinguishing individual $\mathrm{Pt}$ nanospheres electrodeposited on a glassy carbon substrate which catalyze the oxygen reduction reaction (ORR). ${ }^{27}$

The HOR at an individual nitrogen-doped, palladium nanoparticle-modified carbon nanotube (N-CNT-Pd) impacting with a CFE has been monitored voltammetrically. The residence times of the N-CNT-Pds at the electrode surface are considerable (cf. tens of seconds), implying a relatively strong physical contact with the electrode. However, during the course of the impact the Faradaic current fluctuates significantly; these fluctuations were observed as "noise" in current-time measurements. ${ }^{1}$ Consequently, the aim of this work is to identify the physical origins of these fluctuations in the recorded current. This is undertaken by comparing the voltammetric responses of individual N-CNT-Pds toward the HOR at both carbon and gold substrates. The electronic structures of these two materials differ significantly. The carbon fiber used within this work is predominantly graphitic in nature; consequently the material is a semimetal, and the density of states of the two materials at their respective Fermi levels differs by 2 orders of magnitude (0.28 states atom ${ }^{-1} \mathrm{eV}^{-1}$ for $\mathrm{Au}$ vs $2.2 \times 10^{-3}$ states $\mathrm{eV}^{-1}$ atom $^{-1}$ for HOPG). ${ }^{16}$ This difference in electronic structure is reflected accordingly, as well as in the case of the anisotropic resistivity of graphitic carbon. ${ }^{28-30}$ Given that the fluctuations in current likely arise from its modulation at the nanoparticle/ electrode interface, by altering the electronic structure of the electrode the physical origin of this modulation can be probed.

\section{EXPERIMENTAL SECTION}

Chemicals. Nitrogen-doped carbon nanotubes decorated with palladium nanoparticles (N-CNT-Pds) were prepared from carbon nanotubes purchased from Pyrograf Products Inc. (Ohio, USA) using the method described in the work of $\mathrm{Li}$ et al. ${ }^{1}$ The carbon nanotubes (CNTs) were functionalized by oxidation in $\mathrm{HNO}_{3}$ to produce $\mathrm{O}-\mathrm{CNT}$. Nitrogen-containing functional groups were introduced by a flow of $\mathrm{NH}_{3}$ for $4 \mathrm{~h}$ at $600{ }^{\circ} \mathrm{C}$ in a tubular quartz reactor, giving N-CNTs. Pd ions coordinated to these functionalities when added to a solution of the N-CNTs; a hydrolysis treatment was then applied to crystallize the precursors. This realized N-CNT-Pd with a 2.4 wt \% loading of $\mathrm{Pd}$ with a high dispersion scale, as characterized by TEM and SEM in the work of $\mathrm{Li}$ et al. ${ }^{1,31}$

$\mathrm{KNO}_{3}$ was obtained from Sigma-Aldrich and was used as received without further purification. All solutions were made up with deionized water (resistivity $\geq 18.2 \mathrm{M} \Omega \mathrm{cm}$, Millipore) and outgassed with $\mathrm{N}_{2}$ before taking measurements at $298 \mathrm{~K}$. Hydrogen $\left(\geq 99.98 \% \mathrm{H}_{2}\right)$ and nitrogen were supplied by BOC, Surrey, UK.

HOR at Macroelectrodes. The Pt and Au macroelectrodes used in the initial HOR experiment (of diameter 1.6 and 3.0 $\mathrm{mm}$, respectively) were polished with $1,0.3$, then $0.05 \mu \mathrm{m}$ alumina slurry, rinsed with deionized water, and dried with $\mathrm{N}_{2}$ before use.

HOR at a Au Microelectrode. The Au microelectrode (made in-house, diameter $48 \mu \mathrm{m}$ from electrochemical sizing) was polished with the same grades of alumina. It was then polished on a clean, damp polishing pad to remove any embedded alumina residue, before rinsing in deionized water and drying with $\mathrm{N}_{2}$. Experiments on the $\mathrm{Au}$ microelectrode were carried out at $T=298 \pm 1 \mathrm{~K}$ in a thermostated Faraday cage using an Autolab potentiostat (Metrohm-Autolab BV, Netherlands). Electrolyte solutions were outgassed with $\mathrm{N}_{2}$ and $\mathrm{H}_{2}$-saturated by bubbling for $5 \mathrm{~min}$ immediately before scans were recorded.

Nanoimpact Experiments. Nanoimpact experiments on the $\mathrm{Au}$ microelectrode were performed with a two-electrode setup, a Ag wire serving as both a counter and pseudoreference electrode. This facilitated the use of a small volume cell (see $\mathrm{SI}$ ). A suspension of N-CNT-Pd was prepared by sonicating 2.4 $\mathrm{mg}$ of N-CNT-Pd in $5 \mathrm{~mL}$ of $0.2 \mathrm{M} \mathrm{KNO}_{3}$ solution in a Fisher 
Scientific FB15050 ultrasonic bath until the nanoparticles were well-dispersed. A $20 \mu \mathrm{L}$ aliquot of this suspension (containing $9.6 \mu \mathrm{g}$ of N-CNT-Pd) was then added to $180 \mu \mathrm{L}$ of $0.2 \mathrm{M}$ $\mathrm{KNO}_{3}$ electrolyte which had previously been outgassed with $\mathrm{N}_{2}$ and $\mathrm{H}_{2}$-saturated. This diluted suspension was then bubbled quickly $(\sim 5 \mathrm{~s})$ with $\mathrm{H}_{2}$ to disperse the added nanoparticles and kept sealed under a slight positive pressure of $\mathrm{H}_{2}$ as measurements were taken. Higher suspension to electrolyte volume ratios were also used in order to increase the frequency of impact events (giving rise to volumes $>200 \mu \mathrm{L}$ ).

Chronoamperograms were measured at a potential of $+0.6 \mathrm{~V}$ vs Ag wire for $t=30 \mathrm{~s}$. Immediately after a N-CNT-Pd impact (or nonzero current from a successful immersion was observed as described below), a CV was recorded at $50 \mathrm{mV} \mathrm{s}^{-1}$ from -0.8 to $+0.6 \mathrm{~V}$ vs $\mathrm{Ag}$ wire starting at a potential of $-0.775 \mathrm{~V}$, scanning oxidatively first.

Immersion Experiments. Immersion experiments used a conventional three-electrode setup with a mercurous sulfate reference electrode (MSE) supplied by ALS, Japan, and Pt counter electrode. They were conducted by immersing the electrode into the undiluted suspension as prepared above (2.4 $\mathrm{mg}$ of N-CNT-Pd in $5 \mathrm{~mL}$ of $0.2 \mathrm{M} \mathrm{KNO}_{3}$ ) for a few seconds before removal. Over a period of several minutes the remaining solvent on the electrode surface was allowed to evaporate to dryness in air at room temperature. During this time in which the electrode was in contact with the suspension, the stochastic impact of nanotubes occurred leaving nanotubes adsorbed on the electrode surface. The complete drying of the electrode following this adsorption promoted their tighter adhesion. This procedure can be described as a stochastic ex-situ adsorption followed by drying, the use of "stochastic" reflecting the random nature of the impacts and "ex-situ" referring to a modification made outside the electrochemical cell in which measurements were made. Adsorption onto the electrode surface was not successful on every attempt $(\leq 50 \%$ success rate), first due to the comparable sizes of the electrode and the nanotubes and second due to the limited time in which the electrode was in contact with the suspension.

The electrode was then transferred to an aqueous electrochemical cell of $0.2 \mathrm{M} \mathrm{KNO}_{3}$ without the nanotubes of study which had been previously saturated with hydrogen; the electrical contact of any existing nanotubes immobilized on the electrode surface was preserved in this process. Successful adsorptions resulted in an observable HOR signal above the control experiments which were run in the $\mathrm{H}_{2}$-saturated $0.2 \mathrm{M}$ $\mathrm{KNO}_{3}$ without nanotubes and a clean electrode surface.

The chronoamperometric and cyclic voltammetric responses of the $\mathrm{Au}$ microelectrode and the data from nanoimpacts experiments on the carbon microfibre electrodes (prepared as in Ellison et al. ${ }^{32}$ ), adapted from $\mathrm{Li}$ et al., ${ }^{1}$ are included for comparison. Briefly, a larger volume cell with a three-electrode setup was employed with $\mathrm{Ag} / \mathrm{AgCl}(1.0 \mathrm{M} \mathrm{KCl})$ as a reference electrode and $\mathrm{Pt}$ foil as a counter electrode, and chronoamperograms were recorded at $+0.1 \mathrm{~V}$ vs $\mathrm{Ag} / \mathrm{AgCl}(1.0 \mathrm{M} \mathrm{KCl})$.

\section{RESULTS AND DISCUSSION}

The oxidation of a hydrogen-saturated aqueous solution (at a concentration of $0.78 \mathrm{mM}$ ) containing $0.2 \mathrm{M} \mathrm{KNO}_{3}$ was studied voltammetrically at a platinum, gold, and glassy carbon macroelectrode (Figure 1). On the platinum substrate a clear quasi-reversible wave was observed at $-0.4 \mathrm{~V}$ vs $\mathrm{Ag} / \mathrm{AgCl}$ corresponding to the two-electron oxidation of hydrogen, as given by

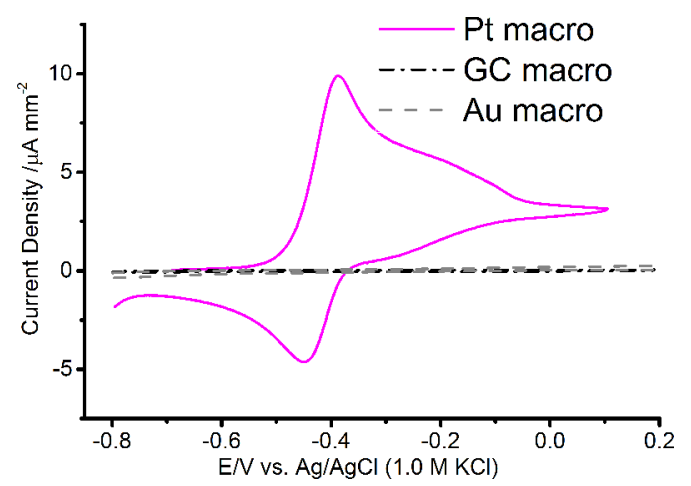

Figure 1. Cyclic voltammetric response of the $\mathrm{HOR}\left(\left[\mathrm{H}_{2}\right]_{\text {sat. }}=0.78\right.$ $\mathrm{mM}$ ) on Pt (pink), Au (gray dashed), and GC (black dot-dashed) macroelectrodes; notably the HOR peak $\sim-0.4 \mathrm{~V}$ (vs $\mathrm{Ag} / \mathrm{AgCl}$ ) is absent on $\mathrm{Au}$ and GC. The response on Pt was recorded against an MSE (+0.64 V vs SHE) reference electrode, so has been shifted by $+0.405 \mathrm{~V}$ for comparison with the $\mathrm{GC}$ and $\mathrm{Au}$ scans taken vs $\mathrm{Ag} / \mathrm{AgCl}$ $(+0.235 \mathrm{~V}$ vs SHE).

$$
\mathrm{H}_{2}-2 \mathrm{e}^{-} \rightarrow 2 \mathrm{H}^{+}
$$

At higher potentials (between -0.3 and $-0.1 \mathrm{~V}$ vs $\mathrm{Ag} / \mathrm{AgCl}$ ) the diffusional tail of the voltammogram is distorted due to the desorption/adsorption of the hydrogen from the platinum surface. ${ }^{33}$ We note that the position of this voltammetric wave is significantly below $0.0 \mathrm{~V}$ (vs SHE). This significant shift in the equilibrium potential for the redox couple arises predominantly from the nonunity stoichiometry of the redox reaction and the fact that under standard conditions ( 1 bar pressure hydrogen) the solution-phase concentration of protons and hydrogen differs by almost 3 orders of magnitude. $^{34}$

In contrast to the voltammetric response recorded at the platinum electrode, the glassy carbon and gold electrodes exhibited no appreciable activity toward the oxidation of hydrogen prior to the breakdown of the solvent. This result is itself of interest due to the fact that both carbon and gold are able to electrochemically reduce protons, albeit at significant overpotentials (see Supporting Information). Beneficially this asymmetry in the redox abilities of the two electrodes toward the hydrogen/proton couple provides two suitable substrates upon which electrocatalysis by suitably decorated nanotubes may be investigated.

In previous work, a CFE was immersed into a hydrogensaturated solution containing N-CNT-Pd. ${ }^{1}$ In the absence of carbon nanotubes no chronoamperometric features beyond the capacitative charging of the electrode were observed. Conversely, in the presence of the carbon nanotubes large steps in the current were observed. Figure 2 (black lines) provides three examples of such features. These net steps in current are ascribed as being due to the arrival of an N-CNT-Pd at the electrode surface where upon impact it is able to catalyze the HOR, hence leading to an increase in the recorded current.

Note that a CFE was employed due to the low diffusion coefficients associated with the carbon nanotubes (cf. $2 \times 10^{-13}$ $\left.\mathrm{m}^{2} \mathrm{~s}^{-1}\right)$. The electrode is microscopic in only one dimension, which significantly increases the probability of an impact occurring by virtue of the attainment of a quasi-steady state diffusion regime. ${ }^{32}$ Unfortunately, such an electrode design is not feasible with the use of gold as the electrode material due to its differing mechanical properties. It should be noted that due to the distinctly smaller size of the gold microelectrode used as 

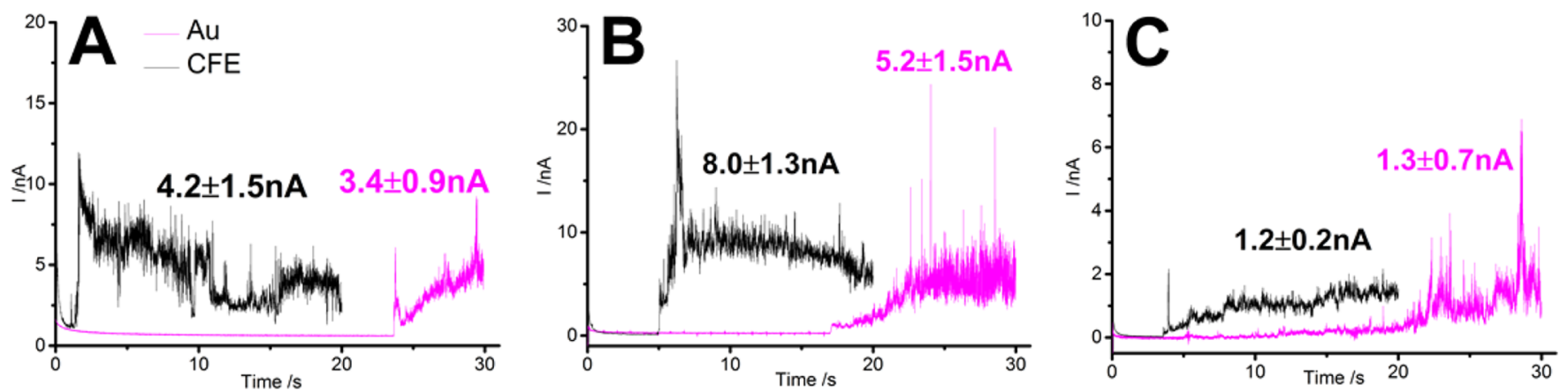

Figure 2. Chronoamperometric profiles showing catalytic oxidative Faradaic steps from impacts of single N-CNT-Pd in $\mathrm{H}_{2}$-sat. $0.2 \mathrm{M} \mathrm{KNO} \mathrm{K}_{3} \mathrm{~N}$ CNT-Pd suspension (impacts with Au microelectrode at $+0.6 \mathrm{~V}$ in pink; impacts with $\mathrm{CFE}$ at $+0.1 \mathrm{~V} \mathrm{vs} \mathrm{Ag} / \mathrm{AgCl}$ in black-both fall in the $I_{\text {ss }}$ range of the HOR). Step height above the background current \pm std. deviation shown. [Impacts on Au in A \& B in 9.6 mg/200 $\mu \mathrm{L}$ (1:9) of suspension; C in $21.6 \mathrm{mg} / 225 \mu \mathrm{L}(1: 5)$ of suspension.] Data recorded at the CFE are adapted with permission from X. Li et al. Single Nanoparticle Voltammetry: Contact Modulation of the Mediated Current. Angew. Chem., Int. Ed., WILEY-VCH Verlag (C 2015 WILEY-VCH Verlag GmbH \& Co. KGaA, Weinheim). ${ }^{1}$
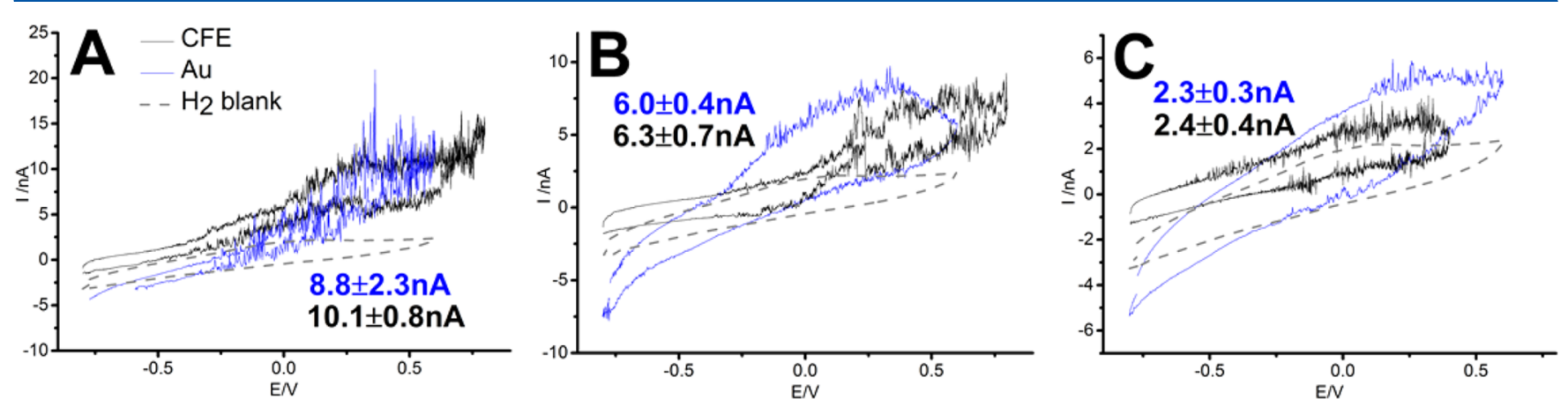

Figure 3. Cyclic voltammetric profiles of $\mathrm{H}_{2}$ oxidation in $0.78 \mathrm{mM}$ suspension, catalyzed by an immobilized N-CNT-Pd on the surface of a Au microelectrode (vs Ag wire, blue) compared to a CFE (vs Ag/AgCl, black). Dashed lines show a blank taken in $\mathrm{H}_{2}$-saturated $0.2 \mathrm{M} \mathrm{KNO}_{3}$ on the $\mathrm{Au}$ microelectrode vs Ag wire, without N-CNT-Pd. $I_{\mathrm{ss}}$ or $I_{\mathrm{qss}}$ above the blank \pm std. deviation shown. [A in $32.64 \mathrm{mg} / 408 \mu \mathrm{L}$ (1:5) of suspension; B \& $\mathrm{C}$ in $54.24 \mathrm{mg} / 453 \mu \mathrm{L}(1: 3)$ of suspension.] Data recorded at the CFE are adapted with permission from X. Li et al. Single Nanoparticle Voltammetry: Contact Modulation of the Mediated Current. Angew. Chem., Int. Ed., WILEY-VCH Verlag (C 2015 WILEY-VCH Verlag GmbH \& Co. KGaA, Weinheim). ${ }^{1}$

compared to the CFE a distinctly higher concentration of solution-phase nanotubes was necessary to offset the decrease in collision probability for a smaller area. Hence, an alternate, small-volume cell design, fully described in the Supporting Information, was required to avoid the use of surplus nanotubes to attain this higher concentration. During the course of an electrocatalytic impact the currents passed are in the nanoampere range, and consequently the use of a two-electrode system is possible in the absence of significant ohmic distortion. The silver wire was therefore used as both a combined pseudoreference and counter electrode.

An oxidizing potential $(0.6 \mathrm{~V}$ vs Ag wire) was applied to the gold working electrode and the resulting current recorded. In the presence of N-CNT-Pd large steps in current were observed as shown in Figure 2 (pink lines). The magnitudes of these steps are, although variable, highly comparable to those recorded for the same nanotube impacts on the carbon fiber electrode. Consequently, these steps in current are again concluded to relate to the arrival of a single nanotube to the electrode surface, where upon contact the palladium present catalyzes the HOR.

Having evidenced the ability to observe the arrival, collision, and electrocatalysis of the HOR at individual carbon nanotubes this work now turns to studying the cyclic voltammetric response of these single entities at a gold microelectrode. This was feasible due to the relatively long residence times of the carbon nanotubes at the electrode surface as evidenced by the durations of the faradaic steps observed in Figure 2 (cf. >10 s). Upon observing an impact of a carbon nanotube to the electrode surface, a cyclic voltammogram of the oxidation of hydrogen at the individual carbon nanotubes was recorded. Figure 3 depicts six examples of the cyclic voltammetric response of individual carbon nanotubes catalyzing the oxidation of the solution-phase hydrogen, three of which were recorded on a carbon fiber electrode and three of which were recorded on a gold microelectrode (as described above). Also depicted in Figure 3 is the response of the gold microelectrode in the absence of N-CNT-Pd. Importantly, as found for the macroelectrode (Figure 1), no peak corresponding to the oxidation of hydrogen was observed on the bare gold surface.

Comparison of the chronoamperometric and voltammetric responses recorded for the single-carbon nanotubes impacting at both the carbon and gold electrodes demonstrates that the electrochemical responses are indistinguishable on the two substrates. This is apparent from Figures 2 and 3 which show fluctuations in the steady-state HOR current, for comparative step heights or steady-state current magnitudes respectively, as standard deviations. Given the extreme differences between the electronic structures of the two electrode materials (described above), this insensitivity to the electrode substrate is of distinct importance. Moreover, the relatively long residence times of 
the carbon nanotubes at the electrode surfaces (cf. tens of seconds) indicate a strong association of the carbon nanotubes to the electrode surface. This result highlights the differences between physical and electrical contact, in that although the nanoparticle is resident at the electrode the properties of the electrical connection are clearly variable with time. The carbon nanotubes are highly conductive due to their multiwalled structure, $^{35}$ and their large sizes preclude current fluctuations relating to a shot noise process arising from the finite nature of the solution-phase hydrogen concentration. ${ }^{36}$ Given this, it is reasonable to conclude that these fluctuations are associated with the electrical connection between the electrode and nanoparticle. Of the cases featuring similar nanoparticle residence times, similar fluctuations have been observed, for example, in the electrochemical response of individual spherical gold nanoparticles catalyzing proton reduction on impact with a carbon fiber electrode. ${ }^{37}$ Consequently, the physical origin of the observed fluctuations is likely common to all the aforementioned systems and is not related to the specific case of impacting carbon nanotubes. Hence, it is credible that the predominant source of the observed current fluctuations relates to the nanoscopic motion of the nanoparticle at the electrode surface which serves to modulate the electrical connection.

In order to evidence this, the article finally turns to considering the response single-carbon nanotubes which were adsorbed at the gold interface and the contact dried prior to electrochemical experimentation. To achieve this, the same gold microelectrode was immersed in the previously described $2.4 \mathrm{mg}$ of N-CNT-Pd/5 mL of $0.2 \mathrm{M} \mathrm{KNO}_{3}$ suspension for a few seconds, removed, and allowed to dry in air. This enabled the stochastic ex-situ adsorption of a nanotube as described above. The modified electrode was then, after drying, inserted into the previously $\mathrm{H}_{2}$-saturated electrolyte. Figure 4 depicts

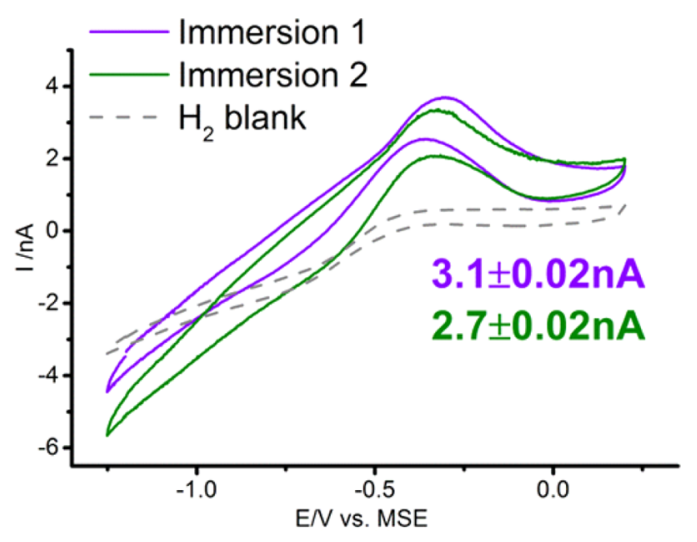

Figure 4. Cyclic voltammetric responses of the HOR from separate immersion experiments on the Au microelectrode (violet, green) as compared to a blank taken in $\mathrm{H}_{2}$-saturated electrolyte (gray dashed). The maximum current before its limitation by Au surface oxidation at higher potentials is labeled \pm std. deviation.

the representative voltammetric responses of the HOR on the $\mathrm{Au}$ microelectrode after modification with N-CNT-Pd. In a majority of cases the current amplitude was comparable to that expected for a single nanotube $(2.75 \mathrm{nA}$ for a single nanotube given an average length of $\left.5 \mu \mathrm{m}^{1}\right)$. The voltammograms are distinctly different from the majority of observed responses of the in-situ impacted electrodes. Notably, the magnitude of the voltammetric wave is not significantly different, suggesting that in both examples a single-carbon nanotube is located on and in electrical contact with the gold electrodes. However, of most importance is that the large current fluctuations are no longer observable and that the HOR is inhibited at higher potentials, although this inhibition is reversible as evidenced by the increase in the current on the reverse scan. This "switching-off" of the HOR at higher overpotentials is almost certainly associated with the electrochemical oxidation of the carbon nanotube-supported palladium nanoparticles. The removal of current fluctuations via drying of the electrode evidences that the noise is not inherent to the electrode material or the nanoparticle but arises from the electrical contact between the nanoparticle and electrode, whereby drying of the electrode improves the contact properties. The extraction of physically meaningful data regarding the kinetics of an electron transfer process has the prerequisite that large fluctuations in the current are not observed during the course of the Faradaic process. Such good electrical contacts may be formed via the drying of the electrode (as demonstrated) or may adventitiously occur over the course of an in situ nanoparticle impact.

\section{CONCLUSIONS}

The chronoamperometric and voltammetric responses of individual N-CNT-Pds have been studied in situ at a gold electrochemical interface and compared to the response observed for impacts at a carbon fiber microwire electrode. Although the electronic properties of the electrode substrates differ significantly, the electrochemical responses are found to be essentially indistinguishable: current fluctuations of a similar magnitude were present for both substrates. Instead the fluctuations are attributed to the nanomotion of the nanotube at the electrode surface, which modulates its electrical contact and therefore the resulting current. Moreover, ex-situ adsorption to the gold electrode followed by drying of the interface improves the electrical connection between the nanoparticle and substrate such that the voltammetric response arising at a single carbon nanotube may be recorded in the absence of large current fluctuations.

\section{ASSOCIATED CONTENT}

\section{Supporting Information}

The Supporting Information is available free of charge on the ACS Publications website at DOI: 10.1021/acs.jpcc.6b00681.

SI Section 1: Setup of the small-volume cell employed for nanoimpact experiments using the Au microelectrode. SI Section 2: Responses of carbon and gold substrates to proton reduction (PDF)

\section{AUTHOR INFORMATION}

\section{Corresponding Author}

*E-mail: richard.compton@chem.ox.ac.uk.

Notes

The authors declare no competing financial interest.

\section{ACKNOWLEDGMENTS}

The research is sponsored by the funding from the European Research Council under the European Union's Seventh Framework Programme (FP/2007-2013)/ERC Grant Agreement n. [320403]. The China Scholarship Council is gratefully acknowledged for funding $\mathrm{X}$. Li's $\mathrm{PhD}$ course. We thank the support from the Shanghai Key Laboratory of Materials Protection and Advanced Materials in Electric Power and the 
National Natural Science Foundation of China (No. 21403137).

\section{REFERENCES}

(1) Li, X.; Batchelor-McAuley, C.; Whitby, S. A. I.; Tschulik, K.; Shao, L.; Compton, R. G. Single Nanoparticle Voltammetry: Contact Modulation of the Mediated Current. Angew. Chem., Int. Ed. 2015, n/a.

(2) Pumera, M. Voltammetry of Carbon Nanotubes and Graphenes: Excitement, Disappointment, and Reality. Chem. Rec. 2012, 12, 201213.

(3) Chen, A.; Ostrom, C. Palladium-Based Nanomaterials: Synthesis and Electrochemical Applications. Chem. Rev. 2015, 115, 1199912044.

(4) Pumera, M. Impact Electrochemistry: Measuring Individual Nanoparticles. ACS Nano 2014, 8, 7555-7558.

(5) Rees, N. V. Electrochemical Insight from Nanoparticle Collisions with Electrodes: A Mini-Review. Electrochem. Commun. 2014, 43, 8386.

(6) Cheng, W.; Compton, R. G. Electrochemical Detection of Nanoparticles by 'Nano-Impact' Methods. TrAC, Trends Anal. Chem. 2014, 58, 79-89.

(7) Zhou, Y.-G.; Rees, N. V.; Compton, R. G. The Electrochemical Detection and Characterization of Silver Nanoparticles in Aqueous Solution. Angew. Chem., Int. Ed. 2011, 50, 4219-4221.

(8) Kwon, S. J.; Fan, F.-R. F.; Bard, A. J. Observing Iridium Oxide $\left(\mathrm{IrO}_{\mathrm{x}}\right)$ Single Nanoparticle Collisions at Ultramicroelectrodes. J. Am. Chem. Soc. 2010, 132, 13165-13167.

(9) Xiao, X.; Pan, S.; Jang, J. S.; Fan, F.-R. F.; Bard, A. J. Single Nanoparticle Electrocatalysis: Effect of Monolayers on Particle and Electrode on Electron Transfer. J. Phys. Chem. C 2009, 113, 1497814982 .

(10) Stuart, E. J. E.; Tschulik, K.; Lowinsohn, D.; Cullen, J. T.; Compton, R. G. Gold Electrodes from Recordable Cds for the Sensitive, Semi-Quantitative Detection of Commercial Silver Nanoparticles in Seawater Media. Sens. Actuators, B 2014, 195, 223-229.

(11) Xiao, X.; Bard, A. J. Observing Single Nanoparticle Collisions at an Ultramicroelectrode by Electrocatalytic Amplification. J. Am. Chem. Soc. 2007, 129, 9610-9612.

(12) Heyrovsky, M.; Jirkovsky, J.; Struplova-Bartackova, M. Polarography and Voltammetry of Aqueous Colloidal $\mathrm{TiO}_{2}$ Solutions. Langmuir 1995, 11, 4300-4308.

(13) Stuart, E. J. E.; Rees, N. V.; Compton, R. G. Particle-Impact Voltammetry: The Reduction of Hydrogen Peroxide at Silver Nanoparticles Impacting a Carbon Electrode. Chem. Phys. Lett. 2012, 531, 94-97.

(14) Kim, J.; Kim, B.-K.; Cho, S. K.; Bard, A. J. Tunneling Ultramicroelectrode: Nanoelectrodes and Nanoparticle Collisions. J. Am. Chem. Soc. 2014, 136, 8173-8176.

(15) Kim, J.; Bard, A. J. Electrodeposition of Single Nm Size Pt Nanoparticles at a Tunneling Ultramicroelectrode and the Determination of Fast Heterogeneous Kinetics for $\mathrm{Ru}\left(\mathrm{NH}_{3}\right)_{6}{ }^{3+}$ Reduction. J. Am. Chem. Soc. 2016, 138, 975.

(16) Royea, W. J.; Hamann, T. W.; Brunschwig, B. S.; Lewis, N. S. A Comparison between Interfacial Electron-Transfer Rate Constants at Metallic and Graphite Electrodes. J. Phys. Chem. B 2006, 110, 1943319442.

(17) Dasari, R.; Robinson, D. A.; Stevenson, K. J. Ultrasensitive Electroanalytical Tool for Detecting, Sizing, and Evaluating the Catalytic Activity of Platinum Nanoparticles. J. Am. Chem. Soc. 2013, $135,570-573$.

(18) Dasari, R.; Tai, K.; Robinson, D. A.; Stevenson, K. J. Electrochemical Monitoring of Single Nanoparticle Collisions at Mercury-Modified Platinum Ultramicroelectrodes. ACS Nano 2014, 8, 4539-4546.

(19) Dasari, R.; Walther, B.; Robinson, D. A.; Stevenson, K. J. Influence of the Redox Indicator Reaction on Single-Nanoparticle Collisions at Mercury- and Bismuth-Modified Pt Ultramicroelectrodes. Langmuir 2013, 29, 15100-15106.
(20) Bartlett, T. R.; Batchelor-McAuley, C.; Tschulik, K.; Jurkschat, K.; Compton, R. G. Metal-Halide Nanoparticle Formation: Electrolytic and Chemical Synthesis of Mercury(I) Chloride Nanoparticles. ChemElectroChem 2015, 2, 522-528.

(21) Simmons, J. G. Generalized Formula for the Electric Tunnel Effect between Similar Electrodes Separated by a Thin Insulating Film. J. Appl. Phys. 1963, 34, 1793-1803.

(22) Sharvin, Y. V. On the Possible Method for Studying Fermi Surfaces. Sov. Phys. JETP 1965, 21, 655-656.

(23) Holm, R.; Holm, E. Electric Contacts: Theory and Application, 4th ed.; Springer-Verlag: Berlin/Heidelberg/New York, 1967; pp 1-55.

(24) Kahk, J. M.; Rees, N. V.; Pillay, J.; Tshikhudo, R.; Vilakazi, S.; Compton, R. G. Electron Transfer Kinetics at Single Nanoparticles. Nano Today 2012, 7, 174-179.

(25) Park, J. H.; Thorgaard, S. N.; Zhang, B.; Bard, A. J. Single Particle Detection by Area Amplification: Single Wall Carbon Nanotube Attachment to a Nanoelectrode. J. Am. Chem. Soc. 2013, $135,5258-5261$.

(26) Nicholson, P. G.; Zhou, S.; Hinds, G.; Wain, A. J.; Turnbull, A. Electrocatalytic Activity Mapping of Model Fuel Cell Catalyst Films Using Scanning Electrochemical Microscopy. Electrochim. Acta 2009, 54, 4525-4533.

(27) O'Connell, M. A.; Wain, A. J. Mapping Electroactivity at Individual Catalytic Nanostructures Using High-Resolution Scanning Electrochemical-Scanning Ion Conductance Microcopy. Anal. Chem. 2014, 86, 12100-12107.

(28) CRC Handbook of Chemistry and Physics; CRC Press: Cleveland, $\mathrm{OH}, 1974$.

(29) Wallace, P. R. The Band Theory of Graphite. Phys. Rev. 1947, $71,622-634$.

(30) Dutta, A. K. Electrical Conductivity of Single Crystals of Graphite. Phys. Rev. 1953, 90, 187-192.

(31) Li, X.; Batchelor-McAuley, C.; Tschulik, K.; Shao, L.; Compton, R. G. Ultra-Small Palladium Nanoparticle Decorated Carbon Nanotubes: Conductivity and Reactivity. ChemPhysChem 2015, 16, 23222325.

(32) Ellison, J.; Batchelor-McAuley, C.; Tschulik, K.; Compton, R. G. The Use of Cylindrical Micro-Wire Electrodes for Nano-Impact Experiments; Facilitating the Sub-Picomolar Detection of Single Nanoparticles. Sens. Actuators, B 2014, 200, 47-52.

(33) Gloaguen, F.; Léger, J. M.; Lamy, C. An Electrochemical Quartz Crystal Microbalance Study of the Hydrogen Underpotential Deposition at a Pt Electrode. J. Electroanal. Chem. 1999, 467, 186192.

(34) Jiao, X.; Batchelor-McAuley, C.; Kätelhön, E.; Ellison, J.; Tschulik, K.; Compton, R. G. The Subtleties of the Reversible Hydrogen Evolution Reaction Arising from the Nonunity Stoichiometry. J. Phys. Chem. C 2015, 119, 9402-9410.

(35) Kim, K.; Jang, D.; Lee, K.; Kang, H.; Yu, B. Y.; Lee, J. I.; Kim, G. $\mathrm{T}$. Influence of Electrical Contacts on the 1/ F Noise in Individual Multi-Walled Carbon Nanotubes. Nanotechnology 2010, 21, 335702.

(36) Kätelhön, E.; Krause, K. J.; Wolfrum, B.; Compton, R. G. How Many Molecules Are Required to Obtain a Steady Faradaic Current from Mediated Electron Transfer at a Single Nanoparticle on a Supporting Surface? ChemPhysChem 2014, 15, 872-875.

(37) Ly, L. S. Y.; Batchelor-McAuley, C.; Tschulik, K.; Kätelhön, E.; Compton, R. G. A Critical Evaluation of the Interpretation of Electrocatalytic Nanoimpacts. J. Phys. Chem. C 2014, 118, 1775617763. 